\title{
La Biología Contemporánea en Clave de Ciencia, Tecnología e Investigación
}

\author{
Claudia Janneth Jaramillo Sánchez ${ }^{1}$
}

${ }^{1}$ Secretaría de Educación del Distrito, Colombia

claxa414@gmail.com

Como citar: Jaramillo, C. (2021). La Biología Contemporánea en Clave de Ciencia, Tecnología e Investigación. EDUCACIÓN Y CIENCIA, (24). e11508. https:// doi.org/10.19053/0120-7105. eyc.2020.24.e11508

\section{Resumen}

Este artículo desarrolla una revisión documental acerca de aspectos relacionados con la configuración de lo vivo y la vida en el presente, a propósito, de los avances en ciencia y tecnología que resultan indispensables para el impulso de la vitalidad y la productividad. El lente analítico empleado es la noción de contraconducta tomada de la caja de herramientas de Michel Foucault, la cual, hace visible qué condiciones posibilitan activar las conductas de los sujetos en relación con lo vivo y la vida. Bajo esta posición, emerge la investigación como práctica de pensamiento contraconductual, eje transversal del texto que, por un lado, permite vislumbrar y reflexionar sobre algunos problemas contemporáneos de la biología y por el otro, evidencia que tanto la DIYbio como el biohacking plantean modos alternativos de aproximarse a la ciencia y la tecnología para problematizar las relaciones que hemos consolidado con el mundo. Estas cuestiones, proponen otras lecturas y modos de relación entre lo vivo y la vida que amplían la mirada sobre la biología y su enseñanza, fundamental para comprender los asuntos contemporáneos de los que somos participes.

Palabras clave: biología, ciencia, tecnología, investigación, enseñanza.

Recibido: $25 / 07 / 2020$ | Revisado: 03/08/2020

Aprobado: 08/12/2020 | Publicado: 31/12/2020 


\title{
Contemporary Biology Understood from Science, Technology and Innovation
}

\begin{abstract}
This article is based on a documentary review of some aspects related to the configuration of the living and life in the present in relation to advances in science and technology, indispensable for the impulse of vitality and productivity. The analytical lens used was the notion of counterbehavior taken from Michel Foucault's toolbox, which makes visible what conditions make possible to activate the behaviors of the subjects in relation to the living and life, among which emerge, research as a practice of counterbehavioral thinking, transversal axis of the text that, on the one hand, allows to glimpse and reflect on some contemporary problems of biology, and the another hand, it shows that both DIYbio and biohacking propose alternative ways of approaching science and technology in order to problematize the relationships we have consolidated with the world. These questions propose other readings and ways of relating the living and life that broaden the gaze on biology and its teaching, which is fundamental to understand the contemporary issues in which we participate.
\end{abstract}

Keywords: science, technology, biology, research, teaching.

\section{Introducción}

Este escrito surge a partir de una investigación de postgrado que plantea diversas perspectivas para repensar la biología y su enseńanza en clave de las relaciones con el arte, donde el propósito es reflexionar sobre las maneras contemporáneas para apropiarnos de lo vivo y la vida como objeto de estudio de la biología; un entramado configurado por múltiples saberes, prácticas y sujetos que pertenecen a distintos lugares, por lo que su evidente interdisciplinariedad ha bastado para que se pueda abordar de maneras insospechadas. Una de las condiciones que permite el diálogo entre estas dos áreas, es la relación entre ciencia y tecnología, a propósito de sus considerables avances vinculados con la innovación y el desarrollo, los cuales, ponen el acento en una serie de cambios que han ido presentado un crecimiento acelerado.

Los nexos entre la ciencia y la tecnología han propiciado que la vida se mapee en clave de la nanotecnología, la biotecnología, las tecnologías de la información y de la comunicación, y las tecnologías cognitivas que probablemente, fundamentarán los avances previstos para el siglo XXI (Colciencias \& CorpoGen, 2006). Estas cuatro tecnologías denotan una relación signada bajo un mismo propósito: mejorar el rendimiento humano, un enunciado predominante en la contemporaneidad. Como lo muestra National Science Foundation [NSF] (2003), es necesario trabajar por la convergencia de las tecnologías Nano-Bio-Info-Cogno [NBIC] que incluyen: la nanotecnología, referida a la escala nanométrica; la biotecnología, concerniente a la aplicación de los preceptos de la ciencia y la ingeniería al uso directo o indirecto de organismos vivos o sus partes en formas naturales o modificadas; la tecnología de la 
información, que abarca sistemas informáticos aplicados, tanto de hardware como de software, involucrando redes y telecomunicaciones; y, la neurociencia cognitiva concerniente al estudio del comportamiento inteligente.

Las tecnologías NBIC pretenden interpelar la constitución de lo vivo y la vida, en aras de su optimización desde diferentes ópticas que convergen hacia la mejoría de las capacidades vitales, partiendo de una mirada holística y global que acude a la unión de puntos comunes para poner en funcionamiento las relaciones que estas tecnologías proponen entre sí. Aun cuando muchos de estos campos se encuentran en desarrollo, es innegable el papel que la tecnología desempeña, incluso, en la vida cotidiana de los sujetos, y las apropiaciones que se hacen de esta a través de dispositivos y herramientas cada vez más familiares.

Dados estos despliegues científicos y tecnológicos importa preguntarse por la enseñanza de la biología en el presente más allá de los contenidos y competencias que se rigen bajo las ciencias naturales, y por la escuela, como lugar de discusión y reflexión sobre los cambios que se afrontan en las maneras de ver y experimentar la vida. De ahí, que las vertientes de la biología contemporánea a las que se hace alusión en el texto a manera de emergencias como la DIYbio y el biohacking, se convierten en condición de posibilidad para ampliar los senderos de la enseńanza, pues aquellas verdades estrictas y necesarias que se han consolidado frente a lo vivo y la vida se han visto modificadas.

Estas prácticas lejos de equipararse con la convergencia del despliegue tecnológico han movilizado al público, para que se apropie y aplique la ciencia y la tecnología en el diario vivir a modo de exploración de la curiosidad, lo cual, permite un acercamiento familiar que surge de iniciativas propias; esa curiosidad que conmociona y apasiona sin tener prescrito de antemano una estructura unidireccional sobre qué hacer, cómo y para qué hacerlo. Ambas expresiones permiten pensar que la biología a propósito de la investigación, amplía cada vez más sus horizontes en el terreno de confluencia por su preocupación o inquietud incesante sobre lo vivo y la vida.

Se pretende entonces problematizar algunos aspectos de la biología contemporánea, así como visibilizar expresiones o vertientes que emanan de esta, a partir de las cuales se plantean otros acercamientos a la ciencia y la tecnología a propósito de la investigación, punto nodal en torno a las reflexiones sobre los modos de constituirnos como sujetos e inquietarnos por las relaciones que configuramos con el mundo, pero también, sobre el papel que desempeña la biología en la escuela teniendo como base la enseńanza. Más allá de establecer juicios de valor o legitimaciones sobre aquello que resulta pertinente y necesario, se trata de visibilizar modos alternativos a las respuestas exactas, sin querer decir que sean mejores o más válidas, más bien, se encuentran latentes en un campo de posibilidades. 


\section{La Noción Metodológica de Contraconducta: Una Mirada al Gobierno de Sí Mismo}

El panorama de este problema se fundamenta en una lectura metodológica orientada fuera de los juicios de valor, la linealidad, los orígenes y las metas. Se toma como base la caja de herramientas de Michel Foucault, no constituyéndose esto en una apología a Foucault, más bien, en una aplicación edificante con un camino abierto a las posibilidades (Veiga-Neto, 1997). Es así, que se pretende vislumbrar aquello que circula en torno a la biología contemporánea, por lo que se consolidó un archivo documental basado en documentos universitarios, blogs, páginas web, páginas institucionales y otros. En consecuencia, la relevancia de todos los escritos es la misma, pues lo que interesa es identificar los enunciados que movilizan los discursos y las prácticas en torno al problema, para esto, se efectuó la desarticulación de dichos textos en una matriz temática que evidencia las regularidades y dispersiones que emergen, a propósito de las relaciones que se hacen visibles con y entre los archivos partiendo del despliegue de las citas.

En este sentido, una lectura discontinua de la matriz visibiliza enunciados como la DIYbio y el biohacking, que toman cada vez más fuerza con la conformación de comunidades virtuales o la realización de eventos que permiten compartir y expresar problematizaciones en torno a lo vivo y la vida de manera colaborativa, sin mediar preocupaciones económicas. Estas iniciativas también plantean un giro en las comprensiones que se tienen sobre la investigación a partir de las construcciones y apropiaciones de la ciencia y la tecnología, dado que se toma distancia de los argumentos contractuales que incitan a configurarnos como ciudadanos responsables de nuestro entorno presente de cara hacia el futuro.

El lente de análisis empleado es la contraconducta, la cual se entiende como la "lucha contra los procedimientos puestos en práctica para conducir a los otros." (Foucault, 2006, p. 238). Esta noción metodológica emerge de la posibilidad de gobernarse a sí mismo, de activar la conducta al poner el acento en maneras novedosas de ser y actuar, de relacionarse con el mundo, lo cual implica franquear la línea de los preceptos instalados en el deber ser y hacer, al incitar el tejido de posibilidades que emergen del gobierno de los otros, se trata quizá, de un gobierno de sí mismo próximo a la creación.

En palabras de Davidson (2012), las contraconductas subvierten las relaciones de fuerza estabilizadas, afectando novedosamente las posibilidades de acción de los otros, de ahí que, algunas manifestaciones de la biología contemporánea como la DIYbio y el biohacking se asumen como contraconductas en la medida que posibilitan acercamientos de distinta índole a los nexos entre ciencia y tecnología, pero aún más, debates y reflexiones sobre las maneras de comprender lo vivo y la vida en la contemporaneidad. La potencia de esta noción tiene que ver con la posibilidad de poner en cuestión el curso presumible de los acontecimientos, así como los juicios 
de valor encarnados socialmente, para dar paso a la conducción propia y a la elección que emana del despliegue de la inquietud, la atención y la mirada puesta en un problema particular.

Los resultados se abordan en tres apartados que destacan otras maneras de suscitar la investigación, pero también, de repensar la enseñanza de lo vivo y la vida. Así, inicialmente se trata la aproximación al abordaje de la biología como problema contemporáneo que más allá de determinar qué es la biología hoy, se preocupa por desentrańar las relaciones que establecemos con nosotros mismos, con los demás y con el mundo. Luego, se plantean algunas vertientes de la biología contemporánea como la DIYbio, que se desmarca de la replicación de prácticas tecnocientíficas para dar apertura a intereses de tipo personal y local en los que media el trabajo colaborativo, así como la exploración y el acercamiento a la investigación. Finalmente, se presenta el biohacking, fundamentado en el deseo de optimizar el cuerpo humano al incitar una forma de vida sana a partir del autocuidado -alimentación, ejercicio, regulación del sueño, entre otras- e incluso, haciendo uso de dispositivos o herramientas tecnológicas.

\section{La Biología como Problema Contemporáneo en Clave de la Investigación}

La alusión a algunas intersecciones contemporáneas entre ciencia y tecnología implica dar enfocarse en uno de sus puntos nodales: la biología, específicamente a la periferia, a propósito de DIYbio -hágalo usted mismo con la biología-, y el biohacking, formas de comprender lo vivo y la vida que se concatenan con la investigación en la medida que instan a reelaborar las problematizaciones que derivan de lo vivo y la vida. Por lo tanto, no se pretende solucionar los problemas que versan sobre la biología, sino reinventar las relaciones entre ciencia y tecnología movilizando los universales sobre los que se cimientan: ¿̨medio o herramienta?, quizá ni lo uno ni lo otro, porque al sustraer las finalidades que apuntan a la innovación y la productividad, es posible comprender que ni ciencia ni tecnología encierran un eminente juicio de valor, más bien, plasman distintos modos de percibir y comprender el mundo, así como de proponer lugares que permitan su discusión.

Esta deliberación no pretende establecer una definición sobre qué viene siendo la biología hoy, a propósito de los adelantos en ciencia y tecnología, sin embargo, el análisis documental deja ver que se reconoce como una de las ciencias más apasionantes en tanto permite descubrir las maravillas que encierra la naturaleza (Universidad de los Andes, s.f.). Como disciplina científica, la biología tiene por objeto los organismos vivos, específicamente, su origen, evolución y propiedades, abarcando un amplio espectro de saberes cuyo estudio mancomunado alberga los fundamentos de la diversidad de la vida a diferentes escalas tanto espaciales como temporales. Pero ¿cómo se configuran relaciones con lo vivo y la vida?¡cómo han cambiado las formas de abordaje de tal objeto? 
Es una biología que emplea técnicas sofisticadas tomadas de la física, la química y las matemáticas. Emplea complejos métodos que permiten secuenciar el $\mathrm{ADN}$; que recurre a técnicas como la bioinformática para determinar la estructura de las proteínas. Sus objetivos no se limitan a la clasificación de los seres vivos por sus características macroscópicas. (Isaza, 2009, párr. 3)

Cabría señalar, ad initium, que una de las principales particularidades de la biología hoy en día es precisamente su carácter transdisciplinario, su necesidad de dialogar y colaborar, en sus desarrollos e investigaciones, con otras ciencias y áreas del conocimiento, pues gran parte de su campo de estudio se encuentra en áreas fronterizas hasta hace poco inéditas, ocultas y, por ende, desatendidas. (Ruiz \& Velázquez, 2014, p. 520)

El archivo consolidado para este texto, destaca tres aspectos emergentes: el posicionamiento de las ciencias naturales a propósito de la transdisciplinariedad, la aparición de áreas como la bioinformática que proporcionan otras herramientas de análisis de lo vivo, y, la transición entre lo molar y lo molecular, siendo este último el nivel predilecto para gobernar la vida. No obstante, es necesario reconocer que la biología configura un campo potencial de exploración, de ahí que, su esencia durante el siglo XXI se fundamenta en la integración de sus disciplinas subyacentes con la física, la química, la informática, las ingenierías y las matemáticas, con el fin de crear una comunidad investigativa que aborde un amplio rango de problemas científicos y sociales en las áreas de alimentación, medio ambiente, salud y energía (National Academy of Sciences, 2009). Entonces, ¿Qué cambios experimenta la biología como terreno de confluencia de diversas disciplinas?

Según Freire (2008), se han cuestionado las comprensiones que en el siglo XXI se tienen sobre la biología como ciencia de lo natural, en tanto, resulta más apropiado reconocerla como la ciencia de lo artificial, al transformarse en una "ciencia del diseño" de sistemas biológicos, ecosistemas y territorios sostenibles, lo cual, ha propiciado el crecimiento exponencial de la información biológica y de tecnologías que favorecen su minería. En efecto, dada su heterogeneidad, esta disciplina se ha posicionado como un motor de cambio que emana molecularmente con los genes, impulsado por los avances en materia de tecnología y disciplinas emergentes.

La biología se constituye en medio de la molecularidad de lo vivo y la vida, en cuanto que sus componentes son reducidos a la información que portan y, que las alternativas para la estetización de la vida de acuerdo con Lipovetsky y Serroy (2015), se hallan en consonancia con la nueva cultura individualista centrada en el hedonismo, el mayor bienestar, la personalización propia en sintonía con la oferta comercial y el super consumo de productos que rinden culto mediante la auto vigilancia, la prevención y la corrección de defectos a un cuerpo "obsesivo, intranquilo, siempre insatisfecho" (p. 294), por lo que, hacer estética la vida tiene que ver con la necesidad de buscar y brindar soluciones optimas al desempeño de los organismos y del ambiente. 
Las revisiones hechas en torno al ambiente dejan ver cuatro problemas contemporáneos: lo vivo, la animalidad, el movimiento y, la vitalidad, con los que se teje una inquietud sobre los modos en que actualmente construimos relaciones de carácter unívoco, pretensioso y utilitarista. Se trata de hacer visible otros modos de interrogar tales vínculos y posibilidades que, más allá de otorgar soluciones precisas, evidencian maneras de proceder distintas. Sobre el primer problema se encuentra que la pregunta por ¿qué es lo vivo? o ¿qué implica estar vivo?, se circunscribe a tres asuntos centrales: auto mantenimiento, reproducción y evolución. Al respecto, Torres (2009), advierte que los organismos vivos son complejos y adaptados a su ambiente, por lo que, se diferencian tajantemente de los objetos inanimados, sin embargo, las comprensiones que circulan sobre lo vivo como elemento biológico están permeadas por su oferta y demanda, es decir, por su mercantilización, lo cual dista de lo que cultural o socialmente se ha considerado como tal (Fuertes, 2014). Pero entonces:

¿Cuál es la naturaleza de lo viviente? ¿Qué hace a los organismos vivientes diferentes y por qué? ¿Es conceptualmente necesario que el fenómeno viviente esté basado en el carbono, o podrían crearse máquinas vivientes artificiales? ... ¿Qué se requiere para explicar la variedad, complejidad y adaptación de los organismos vivientes? ¿Es el proceso de selección natural suficiente o debemos apelar a algún diseńo inteligente? (Torres, 2009, p. 2)

Esta cita deja en evidencia que lo vivo como inquietud permanente de los desarrollos tecnocientíficos, entra en tensión con "el afán por identificar las dicotomías presentes entre orgánico/sintético y natural/artificial." (Bello, s.f., párr. 5). Se podría añadir que, lo vivo goza de una ambivalencia que se engloba bajo la dinámica de la biodiversidad, y a su vez, circula en calidad de piezas y fragmentos, cada uno, con potencial para maximizar la vitalidad. Por otra parte, la animalidad, ha sido objeto de una discusión expresada en términos de lo animal como bioartefactual, teniendo en cuenta los esfuerzos que se han constituido para teorizar y poner en práctica este discurso, a través de políticas y economías emergentes, reflejadas en

las prácticas de domesticación, el acelerado crecimiento de la industria alimentaria, el reemplazo de los animales de trabajo por máquinas, la presencia del animal-objeto en la vestimenta y la taxidermia, la acelerada urbanización del planeta, la investigación científica y cosmética, y tantos otros procesos problematizan las formas en las que se ha teorizado aquello que los animales son. (Artemasciencia, 2015, párr. 1)

Se trata entonces de deconstruir lo animal como paradigma, dejándolo de considerar parte de la maquinaria antropogénica para verlo desde sí, tal problematización permite preguntarse ¿Qué conexiones establecen los humanos con los animales? ¿Con la pérdida progresiva de la naturaleza natural en dónde queda la animalidad? De esta manera, se plantea la dualidad de nuestras relaciones con los animales en tanto compańeros de vida y objetos del mercado, ejemplo de ello es el sacrificio emprendido a través de la alimentación, y el saber técnico científico. 
En el primer caso, se reconoce que los animales ocupan un sitio fundamental en la producción de alimentos, a propósito de la medicina, la inmunología y la salud pública, saberes que permitieron otorgarles criterios de civilidad y progreso; en el segundo, se han desarrollado Organismos Genéticamente Modificados con los que ha sido posible estudiar mecanismos genéticos, enzimáticos y/o protéicos que explican ciertas condiciones adversas, pero a su vez, los argumentos éticos cuestionan tal creación partiendo del hecho que no son naturales, no se respeta su valor intrínseco y por tanto, tienden a convertirse en una amenaza.

La inquietud que se desglosa por la animalidad plantea una mirada histórica a las condiciones de posibilidad que han forjado una brecha entre los humanos y los demás animales, pues las relaciones que se entablan con los últimos como objetos de uso y experimentación conducen a la mejora exclusiva de nuestras condiciones de vida, es así, como la pregunta por la animalidad se vincula con las apropiaciones que hacemos sobre lo vivo y la vida, y, la condición de existencia propia.

En cuanto al movimiento y la vitalidad, se acude a variados campos del saber como el arte, la biología, la biofísica, la biomecánica y la filosofía, para explorar e interrogar las complejas relaciones conceptuales y técnicas entre la vida, el movimiento y las percepciones que se tienen sobre la vitalidad. Estos diálogos interdisciplinares permiten comprender los cambios que experimenta la vida, a partir de las intervenciones que ejercen las ciencias y las tecnologías, junto con la aplicación de los principios de ingeniería, y que convierten los componentes vitales en materia prima susceptible de ser manipulada, así, el movimiento se agencia, cambia, es visceral y está vivo (University Western of Australia, 2013).

Estos problemas aluden a una muestra de la investigación como posibilidad de inquietarnos a nosotros mismos, de prestar atención a lo que pasa y cómo nos pasa, y de congregar al otro y sus apuestas de vida a través de los polos entre los que discurre la vida y lo vivo. Por ello, la biología propicia relaciones consonantes con las verdades que erigen la calidad de vida, retomando la psicologización y sensualización del bienestar que según Lipovetsky y Serroy (2015), entrañan experiencias sensitivas y emocionales fraguadas en el vivir mejor, dado que "se impone una nueva era del bienestar, caracterizada por una demanda cualitativa, cultural y natural de un entorno sensible y armonioso." (p. 339). Pero también, esta ciencia crea nexos resonantes, tejidos con las problematizaciones que se desprenden al asumir la univocidad del vivir más y mejor, y, disonantes, desde las posibilidades de inquietarnos sobre nosotros mismos y las relaciones que entablamos con el mundo, que, entre otros aspectos, son fomentados por la investigación.

Por otro lado, la enseñanza de la biología es un asunto necesario actualmente, dadas las transformaciones que se evidencian a nivel global, pues no solo son susceptibles de problematizarse en la escuela sino en los lugares por donde la sociedad transita. Si bien la biología en clave de la transdisciplinariedad cobra fuerza para solucionar problemas 
urgentes y prioritarios reflejados en sus aplicaciones en áreas como la conservación de la biodiversidad, la reforestación, el mejoramiento de la producción en plantas y animales de interés económico, el aprovechamiento de productos de origen natural para su utilización en agricultura, la industria y la medicina, entre otras, es relevante preguntar ¿Cuáles son las posibilidades de la biología y su enseńanza más allá de la solución imperiosa de los problemas a los que nos vemos abocados como sociedad?

\section{De la DIYbio Hágalo Usted Mismo, Hágalo con Otros y Hágalo con la Biología}

Ahora bien, ¿De qué manera la ciencia y la tecnología se pueden aproximar a la cotidianidad de los sujetos?. La biología DIY otorga algunas pistas sobre modos alternos de entenderlas y ponerlas en práctica pues, "tiene el potencial de proporcionar medios tanto como para repensar la biología moderna y tradicional como para mover la biotecnología fuera del laboratorio y trasladarla a la vida cotidiana de las personas." (Pin, 2014, p. 93) al involucrar garajes, hogares y laboratorios, pero con un manejo diferente al tradicional. Se trata de un movimiento mundial que, según la Red SynbioMX (2014), busca hacer de las ciencias biológicas un espacio accesible para el público, el cual, consiste en el desarrollo con código abierto -software libre- que al elaborar tutoriales para la construcción de equipos y metodologías de laboratorio, tanto económicas como sencillas, conforma comunidades de redes accionadas a distancia para compartir, debatir y crear, en aras de un beneficio personal o local, sin ningún riesgo.

Los proyectos de los grupos DIYBio se enfocan en acercar a los ciudadanos a los conceptos y la investigación en Biología, ofreciéndoles los recursos para construir sus propios materiales, para definir sus propios proyectos y/o para involucrarlos en proyectos de relevancia para su comunidad. (Red SynbioMX, 2014, párr.1)

¿Es la DIYbio un medio de divulgación científico y tecnológico? ¿Puede dar lugar a otros modos de relación con la ciencia y la tecnología?. En palabras de Monasor (2014) "hay algo mágico en un lugar en el que se puede trabajar en cualquier proyecto, sin tener que justificar cuántos millones producirá ... trabajando guiados solamente por la sencilla curiosidad que vio nacer los mayores avances de nuestra especie." (párr. 3). Esta cita deja ver que la curiosidad moviliza la posibilidad de inquietarse por sí mismo, de abrirse paso a la investigación sin tener un fin certero o un compromiso contractual. Por ejemplo, DIYbio.org es una comunidad estadounidense que vincula variedad de grupos locales y extranjeros, los cuales, cuentan con laboratorios tipo "Hágalo Usted Mismo". Estos espacios se encuentran aproximadamente 20 en Norteamérica, 16 en Europa, 2 en Asia y 2 en Australia, donde es posible experimentar con herramientas científicas (El Informador, 2014).

De igual forma, Genspace una organización neoyorquina sin ánimo de lucro, constituida por un colectivo de artistas, ingenieros, biólogos y escritores, dedicada 
a promover la ciencia ciudadana y el acceso a la biotecnología. En 2010 fundó su primer "laboratorio comunitario de biotecnología" donde se ofertan cursos prácticos o experiencias extracurriculares para todo tipo de público, sin requerir formación previa relacionada con el manejo de laboratorio, particularmente, en las áreas de la biología molecular y la biología sintética, pues como laboratorio colectivo se ofrece la posibilidad de trabajar proyectos propios a través de una experiencia netamente inmersiva (Genspace, 2009). Tal experiencia no es costosa, por el contrario, da apertura a posibilidades de fraguar encuentros con las prácticas científicas desde la perspectiva del hágalo usted mismo y hágalo con la biología, que no escinde el hágalo con otros, pues también convoca un trabajo colaborativo.

En Genspace, cualquiera puede trabajar en un laboratorio de biotecnología en el proyecto de su propia elección. Por $\$ 100$ al mes, obtiene acceso 24/7 a las instalaciones de laboratorio de Genspace, equipo y personal voluntario. Proporcionamos muchos suministros comunes, tales como guantes, cajas de petri, y puntas para las pipetas. Usted es responsable de costear cualquier reactivo o equipo para su proyecto, incluyendo material biológico, siempre y cuando cumpla con nuestras directrices de Nivel de Bioseguridad 1. (Genspace, 2009, párr.5)

Es preciso señalar que estos espacios resultan llamativos en la medida que abren la posibilidad para que el público experimente con sus curiosidades, gustos y sensaciones. En este sentido, si bien se incitan experiencias novedosas, no se dan por sentadas ni se conducen, por lo que están más próximas al gobierno de sí mismos a manera de contraconductas, poniendo el acento en las posibilidades de explorar lo vivo y la vida. Así mismo, destaca el hecho de modificar los escenarios de investigación existentes al irrumpirlos, difuminando roles y responsabilidades sociales sin comprometer la permanencia de otros. Según los informes publicados por la Organización de Naciones Unidas y el gobierno estadounidense, el potencial que encierran los colectivos DIYbio es enorme y positivo, aun cuando no se descartan los riesgos, sus principios éticos y modos de proceder son adecuados (Monasor, 2014).

\section{Hágalo Usted Mismo a través del Biohacking}

De acuerdo con algunas revisiones teóricas, es posible identificar una vertiente de la DIYbio: el biohacking, aunque generalmente se establecen como sinónimos, podría precisarse que éste se basa en las prácticas del hágalo usted mismo, pero en asociación con el transhumanismo. De acuerdo con los planteamientos de More (1990), ambos escenarios científicos comparten con el humanismo los principios concretados en la razón, el progreso y los valores centrados en el bienestar, tomando distancia de consideraciones dogmáticas, e incluso, desafiando los límites de lo humano con los avances en ciencia y tecnología, que conjugados con un pensamiento crítico y creativo tienen como objetivo acelerar la transición de la condición humana hacia una transhumana o posthumana. Esta transformación también implica aludir al 
posicionamiento de un ambiente transhumano que genere las condiciones necesarias para mantener una sociedad abierta hacia el progreso constante.

A propósito del biohacking, la perspectiva transhumana se hace visible en tanto gestiona la biología hacia el uso de técnicas nutricionales, médicas y electrónicas con el propósito de mejorar la condición humana, maximizando las capacidades vitales de los sujetos -físicas, mentales y emocionales- (Rodríguez, 2017). Así, a partir de los desarrollos tecnológicos, se pretende familiarizar al público con la nanotecnología, la ingeniería genética y los implantes, siendo estos últimos una opción recurrente para accionar la gestión de la vida a distancia, pues se abordan desde el cuerpo y las relaciones que este constituye con el entorno, lo cual, deriva niveles de comunicación más precisos y útiles para el desenvolvimiento humano.

¿Es factible poner la ciencia y la tecnología al servicio del biohacking? ¿Qué diferencia la investigación en biohacking de la planteada en el enfoque $\mathrm{I}+\mathrm{D}$ ? estos interrogantes sugieren comprender que las aproximaciones del público a las prácticas científicas instan su deslocalización. Según Pascual (2016), el hallarse en correspondencia con la premisa del hágalo usted mismo, abarca desde la fabricación de chips, medicinas e implantes, hasta la posibilidad de insertar estas sustancias y dispositivos en el cuerpo por sí mismos, para "superar una limitación de índole física o mental" (párr. 8), y posiblemente, emocional, pero sin detrimento de la salud, pues "Hacerlo eficazmente requiere conocimiento, tecnología y estar dispuesto a experimentar con uno mismo de forma inteligente." (Luna, 2015, párr. 2). Por ejemplo, se puede indagar sobre un estilo de vida basado en una alimentación optimizada para alcanzar niveles superiores de energía, belleza, longevidad y bienestar, sin que esto implique consagrarle la vida entera al tema, de ahí que, siguiendo a Luna (2015), en la medida que se promueve el acceso y el desarrollo de la ciencia y la tecnología, será posible controlar el ambiente y nuestra propia biología en aras de abrazar formas de biohacking cada vez más exitosas.

Pero¿Hasta qué punto es posible hackearse a sí mismo? ¿Biohacking para irrumpirse a sí mismo, a los otros y a lo otro, en clave de la optimización? Pareciera que el deseo de maximizarse se convierte en una necesidad por satisfacer cuando existe la opción de desarrollar los medios y las herramientas por nuestros propios medios. En este punto la investigación tiene lugar con un propósito centrado en la longevidad del cuerpo humano, al eliminar o minimizar prácticas perjudiciales para la salud física y mental, de manera que, además de conservar el cuerpo, se incita a optimizarlo con la tecnología a modo de ensayo y error, dado que, en la medida de sus avances, las mejoras serán cada vez más considerables.

Como lo indica Pascual (2016), dentro del biohacking emerge una variante denominada grinder, cuyo lema inspirado en el transhumanismo es "Tu mente es software - prográmala, Tu cuerpo es una carcasa - cámbialo, la muerte es una enfermedad - cúrala." (párr. 1). De esta manera, en la lógica de optimizar facultades 
vitales como visión nocturna, comunicación a través de bluetooth o identificación mediante chip, los grinder realizan arriesgadas cirugías que no cuentan con los procedimientos adecuados. En este sentido, tal tendencia no solo transgrede el cuerpo al lesionarlo, también lo hace al experimentar una serie de mejoras que no están "científicamente comprobadas", por lo que se trata de contraconductas que desdibujan el riesgo, pues en lugar de verlo como amenaza, potencia la vida de formas inesperadas.

El cuerpo biológico se hace susceptible de explorar e intervenir con la ciencia y la tecnología a manos propias, dado que, implica un conocimiento exhaustivo tanto de lo natural como de lo artificial que podría maximizar el rendimiento y prolongar la existencia para afrontar las dinámicas de la vida regidas y gestionadas por la productividad. Por un lado, el biohacking sugiere contraconductas que incrementan la vitalidad al modificar prácticas relacionadas con la alimentación, la sexualidad, la educación y la salud, que nos subjetivan en clave del cuidado del cuerpo o, en suma, de la estetización de la vida.

Por otro, la tendencia grinder aborda la posibilidad de considerar que la humanidad puede y debe trascender toda improbabilidad de permanecer activa a lo largo de la vida, de manera, que si no se arriesga o experimenta mediante el lazo científicotecnológico que se vislumbra como condición de oportunidad de la emancipación del cuerpo y, por ende, de la vida, no será viable emprender un giro radical en las maneras de verse, conocerse, sentirse vivo y en definitiva, de estetizarse. Acudiendo así, a otros modos de proceder que aunque no estén avalados científicamente, ponen el acento en la elección personal frente al agotamiento de la vida, especialmente, en un momento en el que la celeridad es constante.

Bien sea como gobierno de sí o como gobierno de los otros, la estetización de la vida funciona como una manija que se moviliza en ambos sentidos, esta pareciera ser una de las verdades que transforman nuestras conductas hacia nuevas maneras de experimentar con nosotros mismos, capaces de aumentar la emancipación competitiva de cara hacia el futuro. Entonces, estetizar la vida es una premisa que funciona en clave tanto de las lógicas que rigen las premisas de la ciencia y la tecnología, como de aquellas contraconductas que permiten rehacer el cuerpo lejos de una belleza estereotipada focalizada en el mejoramiento de las capacidades vitales y la inyección de nuevas conexiones a partir de maniobras centradas en la renovación.

\section{Conclusiones}

Resulta pertinente repensar, analizar y problematizar a profundidad los asuntos contemporáneos de la biología para comprender sus conexiones con otras disciplinas, en clave de la optimización de la vida y, en definitiva, de las relaciones que entablamos con el mundo tanto a nivel global como íntimo. Esto permitiría vislumbrar nuevas betas en la biología y su enseñanza como un gesto primordialmente ético y una 
evocación de la investigación de otro modo, al subvertir el lugar de la biología como disciplina científico-tecnológica y dar apertura a nuevas formas de configurarla, que delaten otras apropiaciones y experimentaciones sobre lo vivo y la vida a manera de inquietud de sí.

Se destaca que, con la ciencia y la tecnología es posible transformar lo que venimos siendo, al habitar tiempos y espacios simultáneos donde todo momento es indicado para producir y rendir, de esta manera, el yo virtual se hace omnipresente. Adicionalmente, la emergencia de nuevos campos que intervienen el nivel molecular de los componentes vitales se implica en el desarrollo de herramientas, procedimientos y técnicas, que permiten la mejora del cuerpo y la mente como un todo capaz de superar la enfermedad y la muerte, incluso, desatándose una de las premisas básicas del transhumanismo. De ahí que, las tecnologías convergentes y la profusión de saberes expertos, inciten una vida activa y realizable que se estetiza en todo sentido.

La DIYbio y el biohacking se proponen como modos alternativos de explorar la ciencia y la tecnología, al propiciar acercamientos fundamentados en la curiosidad, la elección propia, la experimentación, la investigación, en suma, posibilidades que se desmarcan de los requerimientos erigidos por la sociedad, en los que, el desarrollo de la ciencia y la tecnología de la mano de la innovación se traducen en productividad y crecimiento económico. Son modos susceptibles de problematizar la biología y su enseńanza desde una perspectiva disruptiva que propicia la interrogación del saber escolar, las prácticas y los sujetos, que se constituyen en la escuela en constante conexión con las dinámicas contemporáneas del mundo.

Finalmente, la noción metodológica de contraconducta permite ahondar en las oportunidades que como sujetos tenemos para desmarcarnos de las premisas basadas en el deber ser de nuestra vida, es así, como la DIYbio y el biohacking brindan apertura a la exploración individual y colectiva de la ciencia y la tecnología a propósito de la creatividad, el trabajo colaborativo, la posibilidad de comprender y renovar las prácticas científicas y tecnológicas sin detrimento alguno. Estas vertientes se configuran en modos novedosos de implicarse con la biología, así como en nuevas aristas para repensar la enseńanza, las cuales resultan pertinentes para difundir y problematizar en la escuela, pues más allá de otorgarle preponderancia al conocimiento científico, valdría la pena reconocer nuevas formas del saber local y popular que tienen lugar al intentar explorar lo vivo y la vida de diversas maneras que cambian las connotaciones sobre la vida tal cual la conocemos.

\section{Referencias}

Artemasciencia (2015). Lo animal como bioartefactual. https://19573a4f$6099-466$ a-a $84 d-5 b 9 b 5 d 7944 d 3$. filesusr.com/ugd/6923ba_ ba22ae5e543a4fc6ba89cbe4e33d80db.pdf

Bello, M. (s.f.). ¿Qué es VIDA? https://vida.fundaciontelefonica.com/que-es-vida/ 
Colciencias \& CorpoGen. (2006). La biotecnología, motor de desarrollopara la Colombia de 2015.Colciencias. http://repositorio.colciencias.gov.co/handle/11146/553

Davidson, A. (2012). Elogio a la contraconducta (C. Manrique, Trad.). Revista de Estudios Sociales, (43), 152-164. https://doi.org/10.7440/res43.2012.13. (Trabajo original publicado en History of the Human Sciences, 24(4), 25-41.

El Informador (2014, 19 abril). Artistas se alían con científicos para crear obras en Bio Arte. El informador. http://www.informador.com.mx/tecnologia/2014/523925/6/ artistas-se-alian-con-cientificos-para-crear-obras-en-bio-arte.htm

Foucault, M. (2006). Seguridad, territorio y población. Curso en el Collège de France (1977- 1978). Fondo de Cultura Económica.

Freire, J. (2008, 8 julio). El arte desde la biología. http://nomada.blogs.com/ jfreire/2008/07/el-arte-desde-l.html

Fuertes, A. (2014). Bioarte: la otra cara de la ciencia. El Vigía. http://www.elvigia. net/palabra/2016/4/3/bioarte-cara-artistica-ciencia-232865.html

Genspace (2009). New York's Communitiy Lab. LEARN. CREATE. GROW. https:// www.genspace.org/

Isaza, J. (2009, 8 abril). La biología: ciencia del siglo XXI. El Espectador. https://www. elespectador.com/opinion/la-biologia-ciencia-del-siglo-xxi-columna-135062/

Lipovetsky, G., \& Serroy, J. (2015). La estetización del mundo. Vivir en la época del capitalismo artístico. Editorial Anagrama.

Luna, M. (2015, 20 octubre). ¿Qué es el biohacking? https://saludextrema. org/2015/10/28/que-es-el-biohacking/

Monasor, A. (2014, 27 noviembre). El movimiento DIY Bio. http://www.biotekis. es/2014/11/27/que-es-un-biohacker-el-movimiento-diy-bio/

More, M. (1990). Transhumanism: Toward a Futurist Philosophy. Extropy, (6), 6-12. http://fennetic.net/irclextropy/ext6.pdf

National Academy of Sciences (2009). A new biology for the 21st century. https://www. nap.edu/resource/12764/new_biology_final.pdf

National Science Foundation (2003). Converging Technologies for Improving Human Performance. Nanotechnology, Biotechnology, Information Technology and Cognitive Science. http://www.wtec.org/ConvergingTechnologies/Report/NBIC_report. pdf

Pascual, J. (2016, 26 diciembre). Biohacking, el primer paso hacia el transhumanismo. Computer Hoy. http://computerhoy.com/noticias/life/biohacking-primer-pasotranshumanismo-43687 
Pin, P. (2014). Biohacking: Investigación científica como capacidad de performar la realidad. Una revisión transhackfeminista del hackeo de la ciencia. Ritimo 1, 9194. https://www.plateforme-echange.org/IMG/pdf/dossier-st-cast-2014-06-30. pdf

Red SynbioMX (2014, 20 septiembre). DIY Bio México. https://synbiomx.wordpress. com/2014/09/20/diy-bio-mexico/

Rodríguez, P. (2017). Lo + Visto 8: Biohacking, Conviértete en la mejor versión de ti mismo. Fundación Telefónica. https://www.fundaciontelefonica.com/culturadigital/publicaciones/lo-visto-8/627/

Ruiz, R., \& Velázquez, B. (2014). La biología del siglo XXI: Innovación, bioética y bioarte. En M. A. González (Coord.) Pròs bion. Reflexiones naturales sobre arte, ciencia y filosofía (pp. 519-544). Editorial Universidad Nacional Autónoma de México.

Torres, R. (2009). Problemas contemporáneos de la biología. Universidad Pedagógica Nacional.

Universidad de Los Andes. (s.f). Pregrado en Biología. https://cienciasbiologicas. uniandes.edu.co/es/programas/pregrado-biologia

University Western of Australia (2013). SymbioticA-Agency in movement Symposium. http://www.symbiotica.uwa.edu.au/activities/symposiums

Veiga-Neto, A. (1997). Critica post-estructuralista y educación. Editorial Laertes. 